\title{
La notion de Weltanschauung : généalogie d'un concept et d'un processus
}

\author{
ÉLODIE BouBLIL
}

Dans «L'époque des "conceptions du monde" », Heidegger décrit en ces termes l'ambivalence fondamentale et historiale qui parcourt et anime la modernité :

Sans doute les Temps Modernes ont-ils, par suite de l'émancipation de l'homme, amené le règne d'un subjectivisme et d'un individualisme. Mais il est tout aussi certain qu'aucune époque avant les Temps Modernes n'a produit un objectivisme comparable, et qu'en aucune époque précédente le non-individuel n'a eu tant d'importance, sous la forme du collectif. L'essentiel à retenir ici, c'est le jeu nécessaire et réciproque entre subjectivisme et objectivisme. Or, précisément, ce conditionnement renvoie à des processus plus profonds. (115)

Quels sont donc ces «processus plus profonds » dont fait état Heidegger? Quelle est la nature du «conditionnement» résultant de ce jeu, et sous quelles formes culturelles et existentielles se manifesterait-il? Nous souhaiterions montrer qu'une réflexion sur la notion de Weltanschauung, en tant que telle, pourrait permettre d'éclaircir un tel questionnement. À la suite de Dilthey et de Jaspers, Heidegger, en effet, analyse les ressorts ontiques et ontologiques des conceptions ou visions du monde (Weltbilder ou Weltanschauungen) afin d'y puiser une caractérisation phénoménologique et herméneutique du rapport de l'homme moderne à son monde environnant. Selon ces penseurs, la Weltanschauung moderne serait un cadre a priori d'appréhension du réel qui pourrait aliéner la conscience et le monde - les fermer à tout horizon comme les rendre étrangers à eux-mêmes — en même temps qu'elle constituerait paradoxalement le moteur de l'Histoire et définirait les principes du rapport à la Nature. La Weltanschauung est perçue comme 
la résultante de l'idéalisme philosophique qui consacre la toute-puissance du sujet et de ses représentations tout en révélant paradoxalement sa finitude, car il ne peut sortir du cadre de ces dernières. Dès lors, à partir des interprétations de Jaspers et de Heidegger, la Weltanschauung n'apparaît plus que comme un symptôme, voire un qualificatif, qui permet de disqualifier l'époque et ses systèmes intellectuels et politiques. Mais sa quasi disparition du champ philosophique contemporain signale peut-être le caractère polémique de cette notion et la difficulté que comporte le rapport qu'elle introduit — rapport à un monde pourtant difficilement concevable en dehors des déterminations modernes qui le forgent comme tel. Ne faudrait-il pas alors revenir, d'un point de vue phénoménologique, à la chose même qu'est une «vision du monde » afin d'en extraire les possibles implications dans les champs existentiels, anthropologiques et culturels?

Plutôt qu'une typologie des différentes manifestations de la conception du monde moderne ou des applications possibles de la notion, nous souhaiterions dresser une topologie de ses implications dans les champs de la philosophie et de l'existence. Notre travail vise à circonscrire la double nature problématique de la Weltanschauung, en tant qu'elle renvoie à la fois à un concept mais aussi à un processus formateur de ce qu'on pourrait appeler provisoirement un cadre d'existence. Dès lors, l'étude de la notion de Weltanschauung peut permettre de mettre au jour sa prééminence et son actualité, pour penser autant la modernité en tant que telle, que les configurations qui s'y déploient et qui se définissent par rapport à elle, notamment en tant que postmodernité. En effet, nous nous demanderons dans quelle mesure le caractère concurrentiel et pluriel des configurations qui se définissent par rapport à la dynamique moderne constitue une accentuation de leur dépendance à l'égard de celle-ci, et renforce la 
constitution des visions du monde (Weltanschauungen) en images ou cadres du monde (Weltbilder).

Une première définition de la Weltanschauung en tant que concept nous conduira à établir une forme de généalogie des rapports que ce dernier entretient avec les concepts de vérité et de réalité, et à analyser le système eschatologique, ou à tout le moins finaliste, vers lequel il fait signe. Une telle caractérisation nous permettra, dans un deuxième temps, d'envisager la Weltanschauung comme une dynamique constitutive des cadres d'existence moderne et postmoderne, comme un processus qui réclame justement une prise en charge philosophique et conceptuelle avant que ne soit mise en œuvre toute tentative pour le désamorcer. Enfin, nous aborderons les répercussions de cette double nature de la notion de Weltanschauung (à la fois concept et processus) sur le sujet lui-même et la manière dont il a été envisagé, autant d'un point de vue phénoménologique que psychanalytique. Nous verrons que cette notion gagnerait à être réévaluée et repensée à la lumière de l'individu, c'est-à-dire de l'agent proprement dit de la vision. En effet, la définition que l'on donne de ce dernier témoigne de l'influence de l'image moderne du monde, laquelle semble s'inscrire dans un mouvement contraire à l'individuation que pourrait permettre une transformation de son rapport au réel.

\section{Weltanschauung : vérité et réalité}

Dans la modernité, les liens, qui, dans la pensée classique, tenaient ensemble la réflexion et la vie, se cassent. Nous voyons de plus en plus clairement que nous sommes en train de perdre le dénominateur commun de l'expérience de soi-même et de l'expérience du monde. Même l'honorable postulat de la connaissance de soi-même tombe aujourd'hui sous le soupçon d'avoir été naïf; ce qui, jadis, apparaissait comme le sommet de la réflexivité se voit aujourd'hui suspecté d'être peut-être seulement un mirage, né de l'abus de la métaphore de la réflexion. [...] Ainsi, pour la pensée actuelle, intériorités et extériorités, subjectivités et choses se sont séparées pour devenir les unes pour les autres 
des «mondes étrangers ». Ainsi, disparaît en même temps la prémisse classique du philosopher. (Sloterdijk 651)

Les alternatives évoquées, celles de la réflexion et de la vie, de l'homme et du monde, du sujet et de l'objet, et enfin de l'intériorité et de l'extériorité, se sont réalisées selon la dynamique de la métaphysique elle-même, qui a produit les catégories servant à son anéantissement. La description que donne Peter Sloterdijk de la modernité permet de se demander, à la suite de Heidegger, si ce n'est pas «exclusivement une façon moderne de se représenter les choses que de s'enquérir de la "conception du monde" » (Heidegger, «L'époque des "conceptions du monde" » 116), c'est-à-dire en somme de la manière dont le sujet pourrait se représenter ce qu'il nomme le monde. Si les dichotomies réalisées témoignent des apories de la métaphysique classique, la manière de les constater et de les penser reflète la démarche typique de la modernité qui, dans son mouvement nihiliste, incarne le temps et la situation du crépuscule de la pensée spéculative métaphysique. Le mode de configuration de la représentation du monde, la Weltanschauung propre à l'âge moderne des systèmes métaphysiques, semble permettre d'accéder aux raisons d'une telle annihilation.

Analyser la notion de Weltanschauung, de conception ou de vision du monde, invite en premier lieu à évaluer les présupposés qui mettent en jeu le monde, le sujet qui le saisit et le rapport que ce dernier entretient avec la réalité extérieure. En effet, plus qu'un concept opératoire clairement défini, l'idée de Weltanschauung semble appartenir à trois champs conceptuels qu'il faut démêler : celui de la réalité, celui de la vérité et celui de la médiation ou de la déliaison, c'est-à-dire du caractère absolu du principe qui oriente la représentation, voire de la représentation elle-même dans sa dimension totalisante. Ainsi perçue, la Weltanschauung appartiendrait au registre de la métaphysique classique, de l'onto-théologie telle que l'analyse 
Heidegger dans «L'époque des "conceptions du monde" », et correspondrait moins à une réalité qu'à un symptôme paroxystique de l'excès moderne. Encore faut-il interroger alors cette notion à l'aune de l'idée de représentation et de son fonctionnement.

La première occurrence de la notion de Weltanschauung apparait dans l'œuvre critique kantienne, et plus particulièrement au paragraphe 26 la Critique de la faculté de juger. La conception du monde, pour Kant, est apparition (Erscheinung) phénoménale du monde conçu comme ensemble de la réalité empirique. Loin d'avoir une connaissance du monde en tant que totalité, dont l'idée ne peut être donnée au sujet qu'à titre régulateur, le sujet n'appréhende le monde qu'en fonction de la particularité des objets qui le constituent et dont il ne peut nullement saisir l'essence. Évoquer dans ce contexte l'idée de Weltanschauung revient en quelque sorte à formuler une «conception du monde», une représentation régulatrice de ce dernier pour permettre au sujet de s'orienter tant dans le domaine théorique que dans le domaine pratique. L'idée de «monde » dans la philosophie kantienne est associée aux formulations intelligibles et nouménales de la raison pratique. Comme l'écrit Rémi Brague : «Kant réalise de la sorte un découplage capital : l'idée de monde est libérée de la physique. Le monde entre dans le domaine éthique sous la figure du monde intelligible » (236). Mais en se référant toujours à la vérité pour appréhender le réel — que ce soit dans le champ théorique des jugements synthétiques a priori ou dans le champ pratique des idées régulatrices de la raison, constituantes du point de vue de la volonté et de la liberté —, Kant reproduit à son tour un schéma sujet-objet, certes inversé par rapport à la métaphysique antérieure, mais qui ferme encore les rapports du sujet au monde et rend difficilement soutenables les tentatives d'objectivation.

Les développements ultérieurs de la notion de Weltanschaunng - son instrumentalisation comme sa fonction descriptive - paraissent signaler une perversion de 
l'idéalisme critique kantien dont le « découplage » n'aurait servi qu'à donner un nouveau souffle à une eschatologie qu'il convient de circonscrire, et qui remplace un principe transcendant régulateur par un schème causaliste dévoilant une justification, un sens et, de fait, établissant une nouvelle normativité sur un mode immanent. Toute conception du monde évoluerait dans une dichotomie nécessaire, celle du sujet et de l'objet, de la vérité et de la réalité, du rationnel et de l'irrationnel, qui justifie autant l'emprise totalisante qu'elle cherche à avoir sur tous les domaines de l'étant, que la clôture de ce dernier :

Qu'avec ça on rattache la forme au rationnel, et à l'irrationnel la matière, qu'on prenne le rationnel comme le logique et l'irrationnel comme l'illogique, qu'on couple finalement le couple forme-matière avec le couple sujet-objet, et la représentation disposera d'une mécanique conceptuelle à laquelle rien ne saurait désormais plus résister. (Heidegger, «L'origine de l'œuvre d'art » 26)

Les termes employés par Heidegger dans cette assertion révèlent le fonctionnement de la conception moderne du monde et l'accent mis par elle autant sur le mode de la représentation, que sur la «mécanique conceptuelle » d'une raison placée sous l'égide d'un souci d'utilité. Si toute appréhension s'opère alors en fonction d'un intérieur et d'un extérieur, d'une dissociation entre le sujet et l'objet, voire d'une «scission» (Spaltung), pour reprendre le terme de Karl Jaspers $^{1}$, qu'en est-il du monde? Est-il réduit à n'être qu'un «englobant», un support inconnaissable, un cadre, voire une scène sur laquelle évolueraient le sujet totalisant qu'est l'être humain et les objets atomisés et particularisés qui sont à portée de main? Concevoir le monde, n'est-ce pas dès lors élaborer nécessairement une représentation totalisante, indexée sur les critères du vrai et du réel ordonné, et reproduire, par là, le schème métaphysique d'une réalité suprasensible ordonnatrice et justificatrice? Dans son ouvrage intitulé Psychologie der Weltanschauungen, Karl Jaspers décrit la Weltanschauung en ces termes : 
Qu'est-ce qu'une vision du monde? Quelque chose de total et quelque chose d'universel. Une vision du monde n'est pas seulement un savoir, mais elle se révèle dans les méthodes d'évaluation, dans l'organisation de la vie, dans le destin, dans les hiérarchies concrètes des valeurs. Ou pour dire ceci d'une autre manière: quand nous parlons de Weltanschauungen, nous voulons parler des idées de fin et du tout de l'humanité, aussi bien du point de vue subjectif de l'expérience vécue, du pouvoir et de la mentalité, que du point de vue objectif d'un monde façonné tel un objet. (1; nous traduisons)

La Weltanschauung se présente sur le mode de la manifestation, voire de la révélation («offenbart sich ») dans le domaine de la vie et les sphères d'interprétation de celle-ci : valeurs, destinée, organisation. Elle désigne une finalité de l'humanité et non un simple mode contingent de représentation. Par là, l'idée de maîtrise supplante celle de choix, qui réservait encore une place à l'indétermination. Elle possède une valeur subjective puisqu'elle influence autant l'intention que l'intensité mise par le sujet dans son expérience du monde. Son objectivité provient quant à elle d'une figuration (Gestaltung) du monde qui prend la forme d'une réification et d'une constitution de l'objet «monde ». Ainsi, la notion de Weltanschauung révèle à travers sa manifestation une conjonction des concepts de vérité (rapport d'adéquation du sujet à l'objet) et de réalité, puisque la représentation née de la première organise et ordonne l'expérience de la seconde. Mais qu'en est-il de la validité tant épistémologique que métaphysique accordée à ce type de représentation? En s'en tenant aux plans de la justification et de la téléologie, la Weltanschauung, dans ses manifestations modernes, n'est-elle qu'un palliatif au défaut d'ordonnancement du monde dans une époque marquée par le défaut de Dieu? La connexion entre la vérité et la réalité renforce le dispositif d'une représentation objectivante (Vorstellung) qui contribue elle-même, par l'effet d'un redoublement et d'une complexification, aux finalités de la Weltanschauung moderne : la maîtrise de l'être humain et du monde qu'il se construit.

En effet, en en restant à ce principe d'adéquation de la représentation à la chose qu'elle se figure, le sujet recherche l'identification et pose en principe, autant méthodologique que moral, 
une forme d'homogénéisation qui permet de manière réflexive de fonder objectivement l'harmonie et la cohérence d'une représentation du monde totalisante. En constituant le réel rationnellement, toute conception du monde outrepasse le champ délimité des savoirs empiriques spécialisés et réitère en chacun de ceux-ci le principe qui lui est propre : l'identification, devenue attribution d'une fonction inhérente à chaque chose et à chaque manifestation. Les idées de maîtrise et d'homogénéisation sous-tendent la totalisation. Elles désignent le contenu de la notion de Weltanschauung et caractérisent ses manifestations symptomatiques à l'époque moderne. La conception du monde est le fruit d'un esprit systématique qui renforce un dispositif totalisant et unificateur d'inclusion. Un double mouvement de la raison se produit alors avec la modernité : sa formalisation engendre une rationalisation ordonnatrice des éléments constituants du réel, tout en les subsumant sous une vérité elle-même adaptée aux mutations de cette raison, incluse dans une logique finalisée de moyens et de fins. C'est pourquoi, conformément à cette idée, il est possible de traduire ici le terme de Weltanschauung par l'expression «conception du monde ». La raison moderne constitue véritablement le monde en tant que monde, c'est-à-dire en tant qu'ensemble permettant l'investigation théorique et la réalisation de fins pragmatiques. L'articulation de l'investigation et de la réalisation qui est envisagée comme une production de réalités, vise à pallier le chaos d'un monde sans principe transcendant d'ordonnancement.

Dans sa dimension formalisée et formalisatrice, la raison est l'instance autant que l'instrument qui ordonne le réel et organise un «monde ». La redéfinition du logos à l'époque moderne s'émancipe d'une dimension humaine ou divine pour viser une autoconstitution du monde sur un mode objectif. L'idée d'objectivité désigne ici une dépersonnalisation, gage de certitude à l'égard d'un réel défini, dont la circonscription assure la maîtrise. Les méthodes des sciences, marquées par l'idée de recherche et d'investigation, tissent un réseau de plus en plus 
complexe de collections et de connexions, de faits et d'effets, qui enferment le monde dans une conception déterminée. Cette réification de chaque science constituée selon les schèmes d'une raison pragmatiste et productiviste valide le bien-fondé des Weltwissenschaften et invite à penser que ces dernières, loin de s'opposer à une Weltanschauung, sont subordonnées à celle-ci, entendue comme essence dynamique et processuelle de l'Occident moderne. En demandant compte à l'étant «quant à l'étendue de sa disponibilité pour la représentation » (Heidegger, «L'époque des "conceptions du monde" »113), les sciences justifient la dite représentation qui, dans cette réflexivité, se renforce en tant que Weltanschauung, c'est-à-dire en tant qu'image conçue. La vérité redéfinie comme logos qui calcule, évalue et quantifie des probabilités, plus qu'il ne distingue des singularités, se transforme en idéologie, c'est-à-dire en représentation objectivante d'elle-même suivant la logique d'un processus d'uniformisation. Ce dédoublement force le réel en lui appliquant la logique d'une idée, elle-même justifiée par le raisonnement inductif voyant dans le monde l'idée d'une logique. Comment cette formalisation en germe dans la notion de Weltanschaung pourrait-elle être porteuse de valeurs et de sens, tel que le mentionnait Jaspers dans sa définition?

Placées sous l'égide de la maîtrise, les valeurs d'une Weltanschauung sont moins créées que produites et dépendent moins du vouloir, de la volonté entendue comme système de fins, que du pouvoir ${ }^{2}$, de l'effectivité pragmatique du sens qu'elle scelle. Le système de renforcement de la Weltanschauung par le biais de la transformation du logos théorique et pratique assied son emprise sur le réel en tant qu'image fixée et solidifiée. D'un point de vue généalogique, comment évaluer la volonté de puissance sous-jacente à cet ordonnancement? Carl Gustav Jung a étudié les ressorts psychologiques de la Weltanschauung reposant sur des archétypes communs à l'humanité, «c'est-à-dire des systèmes de disponibilité, images et émotions à la fois » (Jung 
41). La Weltanschauung est une forme de réalisation de ces systèmes. Elle détermine à l'égard de la réalité «une attitude exprimée, formée en forme de concepts ». Dès lors il est possible d'envisager une forme de parallélisme entre la vérité et la réalité représentée : la Weltanschauung comme «hypothèse » dévoile sous la forme d'une image conçue ce qu'elle tient pour une «expérience de la vérité de fait $^{3} »(102)$. Cette perspective confirme l'idée d'une subordination des Weltwissenschaften, de la connaissance et de son procès, à l'image conçue comme mise en forme de la réalité, dont le reflet formel assure la validité épistémologique. La question de l'objectivité de la relation au monde est alors le biais par lequel se profile le problème de la constitution des valeurs. En effet, comment ce mode d'objectivation issu d'une raison formalisatrice pourrait-il faire naître un système totalisant de valeurs? De la même façon qu'est duelle la notion de Weltanschauung, à la fois concept théorique et cadre existentiel moderne dans lequel ce concept est formulé, le rapport entre la rationalisation scientifique et les valeurs du monde moderne procède également de ce type de dédoublement. Indexer la réalité sur le principe de vérité et, partant, sur celui de non-contradiction assure cette stabilité tant convoitée par l'idéalisme moderne. L'être, appréhendé et maîtrisé sous la forme d'un arraisonnement du monde par la technologie scientifique, n'est plus dissocié ou comparé à un devoir-être devenu intrinsèque au processus même d'emprise et de maîtrise qui fait de la Weltanschauung moderne un processus de saisissement. Les valeurs et le sens de l'existence surgissent alors de cette finalité intrinsèque en évacuant la foi au profit du savoir. Mais cette attitude, au sens jungien, ne témoigne-t-elle pas elle-même d'une foi, certes sécularisée, mais qui paraît reproduire au plan de la Weltanschauung le schème immanent d'une eschatologie?

Conception et figuration des fins dernières, la Weltanschauung se propose d'assurer au sujet moderne un sens sécuritaire issu de la fixation et de la fixité des objets. Par le caractère 
performatif de la représentation, l'être humain légitime son emprise sur le monde et conçoit son salut sous l'angle d'une expansion et d'une perpétuation de la vie assurées par la technique et la science. Pris dans une circularité herméneutique accentuant d'une certaine manière sa finitude, le sujet croit en l'hypothèse pour la comprendre comme réalité et comprend, au double sens d'une intellection qui est une intégration, la réalité sous la forme de l'image conçue pour croire en la vérité et la légitimité de son attitude. L'expérience de la foi, de la croyance comme Glaubenserfahrung, étudiée par Jaspers (La foi philosophique face à la Révélation 85-103), met en jeu la question de la formulation et de l'adhésion à la Weltanschauung. Cette dernière paraît contenir un principe de perversion de son propre «système de disponibilités ».

En passant de l'image conçue à la conceptualisation du monde, la Weltanschauung accentue le mouvement nihiliste de la métaphysique occidentale en structurant les points de vue en fonction d'une représentation qui est au fond dénuée de valeur, puisque toute finalité réside dans l'«être-produit» lui-même, qui enferme le sujet dans un cercle empêchant tout élargissement de la vision, pourtant condition de possibilité de toute ouverture au monde. La Weltanschauung n'est pas remise en question. Elle est le lieu d'où l'on parle et sur lequel on parle, l'origine et la direction. Elle constitue par ce double mouvement un système eschatologique autosuffisant dont le risque principal réside dans une forme de décollement entre l'image et son contenu éprouvé qui condamnerait, alors, toute velléité intrinsèque de réorientation. L'accentuation de ces processus provoque en effet, selon Jung, un oubli de l'origine véritable de ce système des possibles, à savoir l'inconscient. Or par l'extrapolation de la conscience, la Weltanschauung se décolle du sens et de l'éventuelle légitimité régulatrice de sa formulation pour accéder à une autosuffisance de l'image devenue immédiate et qui corrèle, par là même, la vérité et la réalité. « Nous ne vivons immédiatement que dans le monde des images » 
(Jung 81): «plus est absolue l'influence de la représentation supérieure, plus elle est contraignante, plus elle se comporte comme un complexe autonome se dressant comme une inébranlable réalité en face de la conscience du moi » (87).

Dans cette perspective, la Weltanschauung définit-elle un support qui aide à concevoir le réel et à souffrir de son absence de sens, ou constitue-t-elle un apport transfigurant le monde au rythme du caractère polysémique et polémique de la notion?

\section{Weltanschauung et cadre d'existence}

Réfléchir à la notion de Weltanschauung invite à présent à analyser les conditions de possibilité de son déploiement et à sonder la structure de ses modes de manifestation. Par le langage, elle redouble une dynamique de structuration, celle de sa totalisation en tant que système signifiant, comme celle du réel constitué en «monde» par le sujet parlant. Les composantes du langage, le signe et la signification, sont à la fois le support de la Weltanschauung et les moyens par lesquels elle se surajoute et se superpose au réel à présent configuré. Toute conception du monde en tant que formulation d'une théorie totalisante sur le monde annexe le réel par le langage, en définissant, en fonction d'elle, autant sa substance que ses propriétés. Le monde lui-même n'a de sens en tant que monde qu'une fois constitué comme tel. L'idée de monde résulte ainsi d'une construction autant intellectuelle que langagière qui rive alors toute formulation à l'attitude édifiante du sujet.

Les énoncés de toute Weltanschauung forcent le réel en le structurant en vertu des intentions, finalités d'ordre pragmatique ou pratique, qui guident sa visée projective. Les propositions sur le monde, en tant qu'elles sont contenues dans une conception du monde, renforcent le dispositif de l'image conçue, à mesure qu'elles infèrent une signification du signe 
qui les constitue. Le langage, par le biais d'un discours instituant et législateur fondé sur le principe assimilateur de l'homologie, se fait l'écho du mouvement d'homogénéisation propre à la conceptualisation du monde. La récurrence des signes et la permanence instituée de leurs significations sur un mode diachronique, selon la caractérisation saussurienne, permettent de renforcer le sens global conféré au monde par le sujet de la Weltanschauung. Si le langage se montre à travers la pluralité des formes de discours, il n'en est pas moins rivé à un principe de subordination quant au sens générique de ces derniers. De l'homologie à l'homogénéisation, le discours hiérarchise en son sein les sens qu'il déploie en termes de configuration. Comme le note Merleau-Ponty dans La prose du monde: «Les configurations de notre monde sont toutes changées parce que l'une d'entre elles a été arrachée à sa simple existence pour représenter toutes les autres et devenir clef ou style de ce monde, moyen général de l'interpréter » (184). Le changement remarqué par Merleau-Ponty dans ce passage renvoie à un changement dans la manière de dénoter ou de se référer au monde, mais ne modifie en rien le sens de l'interprétation globale elle-même éclatée auparavant sous la forme d'une appréhension polysémique du monde. Comment envisager la Weltanschauung sous l'angle de la pluralité, ne serait-ce que dans ses modes langagiers de manifestation, alors qu'elle paraît prétendre à une totalisation inclusive, excluant toute altérité et toute alternative? Le discours sur la Weltanschauung n'est-il pas luimême un instrument de son expression, tant la critique pourrait glisser dans une proposition projective alternative?

Eu égard à son rapport au discours, l'expression ou la traduction «conception du monde» doit ainsi être entendue au double sens d'un génitif objectif et subjectif. Elle est conception d'un objet-monde qui trace les frontières de l'existence du sujet et délimite la sphère de son emprise; elle est également conception du monde en tant que sujet constitué s'exprimant 
dans et par le discours de l'individu parlant. La réflexion conduit alors à la question des conditions de possibilité de toute interprétation, voire même de toute élaboration philosophique, tant le cercle du croire et du comprendre (Ricoeur, De l'interprétation 550) paraît se refermer sur les méandres d'une hypostase faisant de toute expression le suppôt et le support de toute tentative de totalisation. Toute formulation sur le monde risque de n'être qu'un succédané de cosmologie dénuée de scientificité ou plutôt usant des sciences pour justifier son horizon en tant que « cosmovision » (Brague 272). Ainsi le discours est-il à la fois produit et productif et invitet-il à penser la question de l'interprétation en revenant à présent à la question des idéologies, de leurs manifestations et de leurs traductions. Penser l'idéologie corrélativement à l'idée de Weltanschauung permet d'insister sur la réification et la logique de cette conception autant dans son sens que dans sa formulation. À travers ce phénomène, il est possible d'examiner la perspective diachronique à l'œuvre dans la dynamique moderne qui transforme le figuré en propre et qui vide les existences individuelles et collectives de leur sens, en rivant celles-ci au support de discours qui leur permettent d'éviter toute remise en question du «je » comme du monde.

Il convient à présent de se pencher sur le phénomène idéologique en tant qu'image réifiée et visant une réalisation qui associerait paradoxalement la vacuité du sens et la solidification de la norme instituée, le nihilisme de la destination et la réification d'un fondement projeté et autoproclamé. Comment l'eschatologie qui meut la Weltanschauung moderne pourrait-elle concilier en son sein une pluralité de discours figurant le monde d'une manière concurrentielle?

L'idéologie force le réel en consacrant l'image et la représentation collective au nom d'un fondement originaire ou d'une justification à venir. Comme l'explique Paul Ricoeur dans $D u$ texte à l'action, l'idéologie possède trois fonctions: la domination, l'intégration et la 
justification. Ces trois modalités d'appréhension et de domestication de la réalité correspondent autant à l'organisation des rapports entre l'individu et la collectivité qu'au procédé intellectuel qui sert le concept lui-même. La fonction d'intégration ou d'inclusion est liée à la représentation objectivante qui donne à voir le monde comme une scène, en exposant des acteurs dont le jeu fournit aux participants la clef de leur identité et leur raison d'être. Le phénomène idéologique est ainsi «lié à la nécessité pour un groupe social de se donner une image de lui-même, de se représenter au sens théâtral du mot, de se mettre en jeu et en scène » (306). La fonction d'intégration recoupe le caractère inclusif du discours en produisant une norme à partir de laquelle le groupe se définit. L'autonomie est fonction d'une emprise sur la réalité polémique originaire et repose sur une assimilation du reflet, de l'image conçue de la société, elle-même convertie en projet. L'analyse de Ricoeur montre la corrélation entre cette assimilation de l'image et sa fonction dissimulatrice. Cette dernière est nécessaire pour ne pas fragmenter autant les actions, devenues sous son influence «événements", que le processus itératif de leur justification. Le «faire » à l'œuvre dans la constitution de l'image est en lui-même projection d'une réalité anesthésiée, c'est-à-dire qui évacue tout obstacle ou souffrance qui impliqueraient une résistance. Dans le creux de cet écart entre le désagrément et le désaccord d'une part, et l'harmonie bien-pensante et unitaire de l'idéologie finalisée de la Weltanschauung d'autre part, se joue un processus de dissimulation qui constitue le monde en tant qu'écran reflétant, à l'instar d'une anamorphose, l'idée que la société souhaite se donner d'elle-même par le biais d'une figuration inscrite de manière sous-jacente dans le pli de la vision. Par cette configuration, l'idéologie est une «obturation du possible» (309), puisque la nécessité à l'œuvre dans la figuration répète la justification d'une fondation originaire et d'un projet qui atteste d'une domination, d'une maitrise autant de l'origine justifiée que de l'à-venir justificateur. Les trois 
fonctions décrites par le philosophe sont donc reliées à un même but eschatologique : la dissimulation par le biais d'une figuration circonscrite et correctrice sert des impératifs biopolitiques de domination qui se donnent comme «projet» dans la projection d'une justification.

Consciente, pourtant, de ce «décollement», la société sous l'emprise de la Weltanschauung ne cesse de rechercher l'adéquation au réel qu'elle se figure comme vrai et légitime, et use par là d'une conception diachronique de la répétition : «L'idéologie est fonction de la distance qui sépare la mémoire sociale d'un avènement qu'il s'agit pourtant de répéter » (307). Au cœur de cette tension, la Weltanschauung se recrée comme téléologie au sein même de l'histoire humaine en instaurant un schème temporel adéquat à son principe de domination : décadence et volonté de retour à l'origine, ou progrès infini d'une raison dont l'absolu pouvoir est pour «demain », l'historicité de la Weltanschauung s'installe paradoxalement, du fait de l'image, dans des utopies et des uchronies qui masquent la réalité de la synchronicité. La Weltanschauung absorbe la dynamique de l'idéologie tout en conservant une réalité qu'elle cherche à transformer, comme elle absorbe la projection de l'utopie qui s'en détache par méfiance et rejet. Ce double mouvement justifie la polysémie et la pluralité des discours qui évoluent en son sein et qui pourtant proviennent d'un langage conçu selon le même format. La pluralité des conceptions du monde constatée empiriquement au cours de l'histoire ne constitue pas un obstacle aux velléités totalisantes de la notion. Tout au contraire, elle atteste l'inscription diachronique de l'intention de ces Weltanschauungen en les faisant évoluer au fil des modifications de la réalité. Les changements apparents de conception ne sont que des variations du reflet perçu qui ne modifient en rien le processus d'explicitation et de justification lié à la projection. Il convient alors à présent de s'interroger sur la notion en tant qu'elle est à la fois un 
support du réel et un apport à celui-ci dans la mesure où, par son inscription dans le temporel, elle influe sur l'évolution des configurations spirituelles de l'époque moderne et se joue d'elles en feignant leur émancipation et leur indépendance à l'égard de toute projection.

Le concept de Weltanschauung appréhendé dans son rapport à l'historicité implique un certain type de position et de posture à l'égard du monde qu'il permet de supporter. En effet, jusqu'à présent, la généalogie de cette notion a montré une ambivalence à l'égard des caractéristiques temporelles de sa justification. Elle est soit orientée vers un avenir développé à partir d'hypothèses et d'archétypes, soit reliée à sa légitimation qui est le fondement même de la rationalité. Autrement dit, la Weltanschauung recèle et scelle à nouveau en son sein une dimension objective et une dimension subjective par-delà la scission apparente qui les met en relation. L'historicité de ce rapport accentue en somme cette dimension en engageant dans cette relation un rapport au monde de l'ordre de la maîtrise ou de la domination. Comme l'a définie Dilthey :

Cette structure est une configuration dans laquelle, sur le fond d'une image (Bild) du monde, se décident les questions du sens et de la signification du monde et dont dérivent l'idéal, le souverain bien [...] cette valorisation de la vie constituant elle-même la couche à partir de laquelle se forment les déterminations de la volonté. (104)

Faut-il dès lors comprendre ou expliquer la Weltanschauung? La comprendre enfermerait peutêtre la subjectivité dans un cercle herméneutique auquel elle prendrait part malgré elle du fait de son insertion parmi les étants mondains; l'expliquer objectiverait son principe et, de manière viciée, donnerait à cette dernière une justification au fondement qu'elle attend. Le concept husserlien d'explicitation (Auslegung), fruit de l'épochè et de la question en retour qui lui est consécutive, peut ouvrir l'analyse autant à un nouvel éclaircissement de la notion qu'à une nouvelle forme de compréhension de son attitude à l'égard du monde. Comme l'indique 
Ricoeur : «Expliciter (Auslegung), c'est déployer le potentiel de sens d'une expérience, ce que Husserl appelle précisément horizons externes et horizons internes de l'objet » (Du texte à l'action 291). Mais «déployer des horizons », externes et internes, poser au dehors le sens que retient l'expérience, invite dans cette optique à analyser les ressorts du monde environnant (Umwelt) et les configurations spirituelles qu'il développe. Questionner le sens reviendrait à examiner les horizons transcendantaux de l'ego, tandis qu'aux horizons de l'objet correspondrait la norme instituée dont ils sont la manifestation. Cependant, la collusion du monde vécu et du monde environnant (Lebenswelt-Umwelt) ne sort pas de la perspective téléologique d'un saisissement du premier par le second, et paraît river toute philosophie de la culture à l'inventaire de ses manifestations. La critique heideggérienne des concepts de Lebenswelt et d'Umwelt et la réintroduction de l'historicité au sein même de la vie du Dasein permettent d'approcher une nouvelle interprétation et d'entrevoir aussi un nouvel écueil au sein de la notion de Weltanschauung. En tant que structuration du réel et assimilation additive de ce dernier, la conception que l'individu a du monde qui l'entoure réifie son existence et la confine dans une « ruinance » caractérisée par un certain «verrouillement ${ }^{4} »($ Abriegelung) qui rejoint le processus de solidification de l'étant étudié précédemment. Pour sortir du monde inauthentique de la facticité et du «on», qui caractérise le Lebenswelt heideggérien, l'individu doit s'éprouver comme Dasein et faire l'expérience du «monde propre » qui seul lui permettrait de s'abstraire d'une Weltanschauung rigide. Dans Les conférences de Cassel (1925), texte antérieur à Être et Temps, Heidegger reprend le questionnement de Dilthey et de Husserl sur la Weltanschauung et la définit en ces termes :

La vision du monde (Weltanschauung) implique une certaine prise de position pratique qui n'est pas seulement momentanée : c'est une attitude permanente à l'égard du monde et de sa propre existence. Elle est formée par l'homme lui-même qui se l'approprie. C'est 
seulement depuis la Renaissance, depuis la liberté à l'égard du lien religieux, que cette possibilité existe. (143)

La Weltanschauung joue le rôle d'un principe d'individuation pour l'être humain détaché du divin et définit à cet égard sa nouvelle prise de position (Stellungsnahme), dans l'ambivalence d'une appropriation trop souvent synonyme d'une prise de possession de l'étant mondain. Ainsi, pour Heidegger, il ne pourrait y avoir de résolution du Dasein et de vie propre qu'à partir d'une compréhension, d'une herméneutique de lui-même, émancipée de la facticité du monde environnant et ce, grâce à l'expérience de l'esseulement. La réflexion que mène Heidegger sur la Weltanschauung est conçue selon une perspective historique et historiale qui exprime les symptômes de l'époque moderne aux prises avec la scission du sujet et de l'objet. Cependant, elle laisse à l'état de question la possibilité d'une communauté, pour l'heure posée comme originaire (Mit-sein) ou bien dénoncée dans la facticité du «on», et qui pourrait permettre d'établir une intersubjectivité - communauté de pensée, médiatisée par un discours dont la réappropriation, l'individualisation serait gage d'individuation.

\section{Weltanschauung et individuation}

Afin de comprendre ce qui se joue dans la Weltanschauung comme mode de saisissement du réel par un sujet lui-même compris par et dans la conception du monde, il faut désormais s'interroger sur le fonctionnement psychique de l'individu et son rapport à la réalité, en tant que celle-ci lui permet de définir son identité et la dynamique de son existence. La psychanalyse freudienne critique elle-même vivement les Weltanschauugen, dans la mesure où ces dernières confortent le sujet dans l'illusion du principe de plaisir et modèlent la culture en vertu d'un désir de remplissement (Wünscherfüllung) du monde, qui pallie le manque originaire de la subjectivité. 
Dans ses Nouvelles conférences sur la psychanalyse, Freud insiste sur la position même de la psychanalyse qu'il érige au rang de science (Weltwissenschaft) par opposition à toute conception du monde qu'il définit de la sorte: «Une Weltanschaunng est une construction intellectuelle, capable de résoudre d'après un unique principe tous les problèmes que pose notre existence. Elle répond ainsi à toutes les questions possibles et permet de ranger à une place déterminée tout ce qui peut nous intéresser»(215). Le principe téléologique et la relative clôture du monde mentionnés par Freud renvoient à la problématique même du sens et de la situation de l'individu dans le monde. Par l'idée d'assignation et le refus de toute conception envisagée telle une intuition (Anschauung), Freud écarte l'idée d'une programmation et d'une planification de l'existence, mais, par ce rejet, ôte au «projet» la possibilité d'une reconversion à titre individuel, dissociée de l'idée de prévision. Qu'en est-il du devenir psychique de la personnalité si cette dernière est réduite à la forclusion devant un principe organisateur et finalisé qui paraît réduire à néant toute velléité de choix et de résolution?

En associant a priori la réalité à une forme de déterminité, la Weltanschauung annihile autant l'espace, en bannissant de son champ d'appréhension les concepts de «proche » et de «lointain », que le temps, puisqu'elle place tout objet sur le mode de l'immédiateté de l'affect désirant définit comme réel. Dans sa définition même, la Weltanschauung s'oppose selon Freud à la science, puisque par la clôture qu'elle instaure, elle empêche le processus de l'investigation et de la recherche qui paraît présupposer une ouverture. De plus, Freud met la psychanalyse sous la tutelle de la vérité et, partant, des principes de non-contradiction et d'adéquation à la réalité extérieure intellectualisée par le sujet analysant. Mais comme Heidegger l'analyse dans «L'époque des "conceptions du monde" », cette dynamique même de la recherche induit une réification et une emprise sur l'étant similaire à la dimension de clôture dénoncée par Freud. 
Selon le fondateur de la psychanalyse, cette dernière n'est pas une Weltanschauung, puisqu'à la différence de la philosophie, elle ne se concentre pas sur le monde, en dessinant son image, mais sur le sujet et le processus de ses représentations. Cependant, si Freud érige la psychanalyse au titre de science différenciée et indépendante de toute Weltanschauung pour cette raison, il n'est pas moins confronté à la nécessité de penser le sujet comme un être-dans-le-monde et, partant, de redéfinir lui-même sa situation en repensant les relations entre subjectivité et réalité. En reconduisant l'idée d'une représentation objectivante (Vorstellung), et en pensant la culture et les institutions des êtres humains dans leur rapport avec ce que Freud nomme le «principe de réalité », la psychanalyse paraît échouer, par cette rationalisation de la situation de l'être humain, à pourvoir l'existence de l'individu d'un sens indépendamment de toute formulation sur le monde, et du principe même de la Weltanschauung moderne : dialectique de la réalisation et de l'interdit, la réalité n'est finalement conçue par Freud que comme un principe extérieur frustrant les désirs polymorphes d'une subjectivité marquée de fait par le refoulement. La culture n'est que la manifestation du sur-moi et la réalité apparaît alors comme un principe de réification de l'étant. La condamnation freudienne à l'endroit de la religion et du mythe conçus comme Weltanschauungen, et discrédités de ce fait, sous-tend un tel processus de rationalisation qui, pourtant, est le fait même de la Weltanschauung de la modernité. Comme le remarque Sloterdijk à propos de la rationalisation freudienne : «Le rationnel apparait comme le couvercle qui couvre l'irrationalité privée et collective » (80). Par cette théorisation de la réalité qui, dans son principe, se veut scientifique et dénuée de présupposé sur le monde, Freud paraît accentuer le clivage sujet/objet et la dynamique de la Weltanschauung moderne qui culmine dans le développement parallèle et concurrent d'un «subjectivisme » et d'un «objectivisme» (Heidegger, «L'époque des "conceptions du monde" »115) paroxystique, aux antipodes de toute individuation réelle. 
Dès lors, comment envisager, à partir du processus de formation de la conception du monde ellemême, c'est-à-dire à partir du fonctionnement psychique du sujet, la possibilité du déploiement d'une individuation et celle d'un sens puisé dans l'expérience du monde?

Il convient de repenser à cet égard les rapports entre le sujet et l'extériorité sociale qui définit son monde environnant. En revenant aux racines affectives de la psyché et à la nécessité primordiale et originaire des archétypes pour cette dernière, la conception jungienne de la Weltanschauung permet de réexaminer, au regard du danger de la rationalisation, le rapport de sens qui s'établit entre le sujet et le monde. Jung émet l'hypothèse d'une dialectique du moi et de l'inconscient, et pose l'idée d'une interaction entre les représentations collectives et la formation individuelle de la personnalité. Le monde en tant qu'ensemble d'images conçues, de représentations, permettrait à l'individu de se définir et de se construire pour autant que ces images ne deviennent pas des «-ismes ». Ainsi, Jung atteste de sa conscience du danger inhérent à toute solidification, étant entendu que les principes figurés ou les types dont parle le psychologue des profondeurs doivent être rattachés à la vie. Afin d'éviter la rigidité d'une individualité centrée sur le moi, il importe de procéder grâce à ces représentations archétypiques à une individuation du Soi, au moyen d'une nécessaire distanciation à l'égard de ces «grandes images ». Seul ce processus permet à l'individu de s'accomplir et de donner un sens et une visée à son existence dans le monde. La psychologie analytique, à titre de science, permet de « réparer » et d'«améliorer » une Weltanschauung devenue représentation singulière et propre à l'individu en cure. La manière même de se représenter le monde est ainsi en jeu dans la construction de l'identité et de l'individualité, et il est important de constater la réciprocité qui s'instaure alors entre le sujet et l'objet de la représentation. Ce pôle qu'est le monde est luimême sujet, puisqu'il vit et agit à travers les représentations dynamiques figurées par les 
archétypes. Cette rétroactivité du monde sur le sujet de la représentation, telle que la pense Jung, n'est pourtant pas l'indice d'une clôture, puisqu'au contraire, elle garde une ouverture pour une individuation entendue comme processus de résolution non rivé à la proximité de la représentation. Si l'image du monde est nécessaire, c'est-à-dire si elle ne peut pas ne pas être l'instance qui médiatise le rapport du sujet au monde, il convient de s'en dissocier tant le poids de l'imitation et de la volonté d'adéquation pèse sur les initiatives du sujet. Le sens de l'existence, à titre individuel, provient d'une libération de l'horizon qui s'obtient par un processus de différenciation et qui implique de fait une nouvelle manière de voir le monde. La Weltanschauung doit donc se défaire de l'idée de «conception » et de construction intellectuelle du monde pour libérer le potentiel heuristique et créateur de l'image, quant à la connaissance que le sujet peut avoir de lui-même. Le sujet peut ainsi édifier une «nouvelle Weltanschauung » à l'aune de son processus d'individuation (Jung 126).

Réciprocité du spéculaire et de la spéculation, de l'image du monde et de la réflexion sur soi, la Weltanschauung, une fois individualisée, retrouverait sa fécondité régulatrice au seuil d'un processus d'individuation nécessitant la monstration (Darstellung) pour s'accomplir dans une création continuée de soi. La position de Jung permet à présent de dissocier, au cœur même de l'image, sa dimension mimétique de sa dimension poḯtique.

La Weltanschauung doit donc être distinguée du Weltbild qui reproduit de manière anthropomorphique la logique du moi et non la dynamique synthétique du Soi. Libéré d'un schème logique et ontologique d'adéquation, l'individu qui saisit le reflet de l'image de son monde sort du mode de l'accaparement (Benommenheit) propre à l'animalité, pour devenir l'être qui se configure un monde, en libérant de nouveaux horizons. Il est alors possible de transformer la notion de Weltanschauung, à partir de la réflexion de Heidegger sur les conceptions du monde, 
en exploitant un autre sens, certes dissimulé jusqu'alors. Heidegger paraît en effet dans «L'époque des "conceptions du monde" » entremêler les notions de Weltanschauung et de Weltbild, et les définit de la sorte :

Image (Bild) désigne donc ici non un simple décalque, mais ce qui se fait entendre dans la tournure allemande : «wir sind über etwas im Bilde » (mot à mot : «nous sommes, quant à quelque chose, dans l'image », c'est-à-dire «nous sommes au fait de cette chose »). Ce qui veut dire: l'affaire en question est telle que nous savons précisément de quoi il retourne. Se faire idée de quelque chose de façon à être fixé, c'est donc placer l'étant luimême devant soi pour voir de quoi il s'agit, et l'ayant ainsi fixé, le maintenir constamment dans cette représentation. (116-117)

Dans l'optique du Weltbild, l'image est fixée et formée en fonction d'une subjectivité égotique qui reproduit le Même à partir d'une détermination préconçue du sens qu'elle cherche illusoirement à trouver dans cette représentation. Loin de laisser libre cours à une circulation du sens, le sujet se veut «au courant » de l'état des choses qu'il re-présente, selon le schéma d'une répétition devenue acquiescement à sa «volonté de puissance», selon la terminologie nietzschéenne. À la différence de l'intuition (Anschauung) qui pourrait viser le monde sans pour autant fixer le principe de sa transitivité, l'image objectivante cible la constitution d'une culture (Bildung) dont la fausse émancipation (Befreiung) n'est que l'envers d'une représentation de l'étant où œuvre la technique.

La «nouvelle Weltanschauung» assume son statut de «construction». Si elle est «image », elle est cependant vue dans un «miroir» qui redouble et dédouble le statut de « copie » pour insérer la différence du «créer » au sein même de l'identité relative du représenté. Dans cette optique, la Weltanschauung se dissocie du Weltbild et pourrait bien relever de «l'être-œuvre» et non plus de «l'être-produit»: «Être-œuvre signifie donc installer un monde» (Heidegger, «L'origine de l'œuvre d'art» 47). Celui-ci s'ordonne comme tel («Welt weltet») sans pour autant être un cadre figuré, puisque l'activité du reflet et les tonalités de 
l'image, associées à une individuation entendue en un sens dynamique, empêchent d'envisager alors le monde comme un support confirmé par les apports rationnels et ratiocinants d'une subjectivité qui n'a plus lieu d'être.

Si la configuration du monde possède une importance du point de vue du processus d'individuation, alors se pose la question du statut de la représentation qui ne serait plus «représentation représentante » mais «représentation réelle ». Une nouvelle Weltanschauung, loin de réduire à néant toute possibilité de sens et d'orientation (Sinn), pourrait être porteuse d'un horizon. L'horizon de sens de l'individu approuve son origine singulière pour envisager son rapport au monde sur le mode, non du relativisme, mais de la relation constituante qui se met en place et s'installe grâce à la vision. Libéré d'un principe d'objectivation, l'horizon des possibles d'une Weltanschauung singulière prendrait acte du réel-monde sans l'indexer sur un principe de non-contradiction.

\section{Conclusion}

Le problème vrai tient à ce que la philosophie perdrait son sens si elle renonçait à juger du présent. Exactement comme une moralité qui serait «interminable et transfinie par principe » ne serait plus moralité, une philosophie qui renoncerait par principe à toute prise de position dans le présent ne serait plus philosophie. Seulement, le fait est qu'en voulant faire face aux problèmes actuels [...] les philosophes de la Weltanschauung manquent tout : ils ne peuvent apporter dans la solution de ces problèmes plus de rigueur que les autres hommes puisqu'ils sont, comme eux, dans la Weltanschauung et n'ont pas de Weltwissenschaft, et pendant qu'ils s'épuisent à penser le présent, ils dérobent à la vraie philosophie le dévouement inconditionné qu'elle exige. Or, une fois constituée, elle permettrait de penser le présent aussi bien que le passé et l'éternel. Aller droit au présent, c'est donc lâcher le solide pour l'illusoire... (Merleau-Ponty, Éloge de la philosophie et autres essais 107)

Cette analyse de Merleau-Ponty montre à quel point la question de la Weltanschauung, comme notion et comme monde environnant (Umwelt), touche la philosophie dans ses 
fondements mêmes et dans le rapport qu'elle tente d'instituer entre l'être humain et le monde. Effectivement, philosopher sur la Weltanschauung revient inévitablement dès lors à philosopher à partir d'elle. Mais en dissociant la configuration propre à la modernité (Weltbild) d'une vision du monde (Weltanschauung) qui configure des possibles et n'arrête pas les horizons ouverts d'un Dasein individué, la philosophie pourrait s'émanciper d'une qualification péjorative de la Weltanschauung qui, dans son opposition à une connaissance du monde (Weltwissenschaft), atteste des exigences du Weltbild. Ainsi, la formule de Merleau-Ponty pourrait être renversée : «Lâcher le solide pour l'illusoire » constituerait moins une erreur qu'une nouvelle prise de conscience.

Délaisser la réification et l'objectivation du monde et comprendre que toute vision est interprétation et, partant, pensée sous l'angle éphémère et singulier de sa condition permettraient autant une mise à distance des configurations symboliques qu'une prise au sérieux de leur jeu, une fois accepté comme tel. Il ne s'agirait donc plus d'ignorer le présent au nom de sa contingence, mais de le laisser advenir en respectant la multiplicité des Weltanschauungen, désormais possibles dans leur pluralité dès lors qu'elles sont dissociées du Weltbild. L'image moderne du monde repose sur les postulats de la métaphysique idéaliste qui confère au sujet une toute-puissance vis-à-vis des choses qu'il constitue comme objets. La rationalisation, l'homogénéisation et l'objectivation à l'œuvre dans la manière moderne d'agencer le monde, confortent l'hypothèse selon laquelle la Weltanschauung pourrait être définie comme une tentative de totalisation du réel. Mais loin de n'être qu'une conception qui accentuerait la séparation d'un «intérieur » et d'un «extérieur», la Weltanschauung, parce qu'elle figure et ouvre un monde à l'homme, a également partie liée avec la formation de la personnalité de ce dernier, qui passe par la médiation de la vision. L'individuation est un processus de confrontation 
au réel et d'incorporation de ce dernier. L'expérience du monde permet alors d'encourager cette dynamique de réalisation.

En étant producteur de réel, le processus d'individuation permis par le regard crée également du sens. Dans cette configuration et dans cette donation de sens se recrée la possibilité même d'un espace commun. Ainsi, si la philosophie est une manière d'interpréter le monde en liant son herméneutique à l'existence, elle peut participer de cette création de sens qui ne réduirait pas son activité à l'inventaire des apories et des échecs de ses postulats fondateurs. La notion de Weltanschauung montre alors, une fois redéfinie, sa pertinence à l'égard du présent du monde comme de l'avenir des hommes. Dans cette réévaluation se profile peut-être à nouveau la légitimité d'un type phénoménologique de philosopher qui ne chercherait pas à ordonner et à classer, mais à montrer et à comprendre. Voir le monde en ce sens, n'est-ce pas amorcer la transformation de ce dernier? Hypothèse qui confirmerait la dimension politique et polémique de la notion de Weltanschauung autant qu'elle expliquerait sa disqualification.

\section{Notes}

${ }^{1}$ «Quel est donc le sens de ce mystère impliqué à tout instant par la scission sujet-objet (SubjektObjekt Spaltung)? Manifestement, c'est que l'être en tant que totalité ne peut être ni objet ni sujet, mais qu'il doit être l' «englobant » qui se manifeste dans cette scission », écrit Karl Jaspers dans son Introduction à la philosophie (29).

${ }^{2}$ Comme l'écrit Sloterdijk au sujet de la caractérisation moderne selon laquelle «savoir c'est pouvoir»: «le moi est le résultat de programmations. Il se forme dans les dressages émotionnels, pratiques, moraux et politiques » (Critique de la raison cynique 91).

${ }^{3}$ « Nous ne serions pas même capables de fixer une hypothèse pour en déduire les conséquences, si nous ne commencions par la tenir pour vraie. Une hypothèse est ce que l'on suppose vrai, et la pensée hypothétique présuppose une expérience de la vérité de fait [...] C'est ainsi que je saisis l'essence concrète du triangle, qui n'est pas un ensemble de "caractères" objectifs, mais la 
formule d'une attitude, une certaine modalité de ma prise sur le monde, une structure », écrit Merleau-Ponty dans son analyse du cogito (Phénoménologie de la perception 442), qui peut être rapprochée de l'interprétation jungienne du mode d'être-au-monde.

4 «Pour dire cette mobilité de la vie qui se fuit elle-même, le cours de 1921-1922 forge les concepts de reluisance, de préstruction et de ruinance. [...] "Nous désignons par reluisance", écrit Heidegger, "le mouvement de la vie en tant qu'il est orienté vers celle-ci", et ce mouvement est préstructif en tant qu'il vise à sécuriser la vie contre l'inquiétude qui l'habite en objectivant celle-ci aussi bien pratiquement que théoriquement, c'est-à-dire en produisant un monde d'objets, en construisant des systèmes de croyances ou de pensée. La vie évite ainsi de se voir elle-même, se fuit, se durcit ou se verrouille elle-même; ce verrouillement (Abriegelung) caractérise la ruinance. Le chemin de la facticité à l'idéal donnant sa cohérence à la vie devient le mouvement de dissimulation qui conduit la vie à se verrouiller elle-même », rapporte J.-C. Gens (introduction aux Conférences de Cassel 63).

\section{Ouvrages cités}

Brague, Rémi. La sagesse du monde. Paris: Le livre de poche, 2002.

Dilthey, Wilhelm. Théorie des conceptions du monde. Trad. L. Sauzin. Paris: P.U.F., 1946.

Freud, Sigmund. Nouvelles conférences sur la psychanalyse. Trad. A. Berman. Paris: Gallimard, 1981.

Heidegger, Martin. Les conférences de Cassel. Trad. et intro. J.-C. Gens. Paris: Vrin, 2003.

—. «L'origine de l'œuvre d'art » et «L'époque des “conceptions du monde” ». Dans Heidegger. Chemins qui ne mènent nulle part. Trad. W. Brokmeier. Paris: Gallimard, 2001.

Jaspers, Karl. La foi philosophique face à la Révélation. Trad. J. Hersch. Paris : Plon, 1953.

—. Introduction à la philosophie. Trad. J. Hersch. Paris: 10/18, 2003.

—. Psychologie der Weltanschauungen. Berlin: Springer Verlag, 1990.

Jung, Carl-Gustav. Problèmes de l'âme moderne. Trad. Y. Le Lay. Paris: Buchet-Chastel, 1984.

Merleau-Ponty, Maurice. Phénoménologie de la perception. Paris: Gallimard, 2002.

—. La prose du monde. Paris: Gallimard, 1997. 
—. Éloge de la philosophie et autres essais. Paris: Gallimard, 2000.

Ricoeur, Paul. De l'interprétation. Essai sur Freud. Paris: Seuil (Points), 2001.

—. Du texte à l'action. Paris: Seuil, 1986.

Sloterdijk Peter. Critique de la raison cynique. Trad. H. Hildenbrand. Paris: Christian Bourgois, 1987. 